\title{
Estudio de las propiedades mecánicas y tribológicas de recubrimientos multicapa de espaciado sub-micrométrico
}

\author{
R.J. RODRÍGUEZ, A. MEDRANO, M. RICO, B. LERGA, R. MARTíNEZ, R. BUENO, J. A. GARCíA \\ Centro de Ingeniería Avanzada de Superficies - AIN, 31191 Cordovilla-Pamplona, Spain
}

\begin{abstract}
El presente trabajo recoge un estudio comparativo de las propiedades mecánicas y tribológicas (microdureza, fluencia, módulo elástico, coeficientes de fricción y desgaste), de multicapas TiN/AlTiN y TiN/CrN depositadas mediante técnicas industriales de PVD. Las capas, de espesores comprendidos entre $25 \mathrm{~nm}$ y $100 \mathrm{~nm}$, se depositaron secuencialmente sobre substratos de WC, hasta obtener un espesor total de unas 2 micras. El estudio mecánico y tribológico comparativo incluyó ensayos de microdureza a cargas entre $10 \mathrm{mN}$ y $750 \mathrm{mN}$, módulo elástico, comportamiento frente a fluencia y coeficientes de fricción y desgaste.
\end{abstract}

Palabras clave:

\section{Study of mechanical and tribological properties of multilayered coatings with sub-micron spacing}

This work gathers a comparative study of the mechanical and tribological behaviour (microhardness under load, Young modulus, elasticplastic properties, friction and wear coefficients) of TiN/CrN and TiN/AlTiN multilayers deposited by conventional industrial PVD techniques. PVD multilayers with about between $50 \mathrm{~nm}$ individual layer thickness were deposited on flat samples of Co-cemented WC achieving a total thickness of about 2 microns. Mechanical and Tribological tests include microhardness under load at final loads between 10 $\mathrm{mN}$ aand $750 \mathrm{mN}$, Young modulus calculations and friction and wear tests.

Keywords: PVD, multilayers, tribology, microhardness, wear, friction.

\section{INTRODUCCIÓN}

En las últimas dos décadas la aplicación industrial de los recubrimientos por PVD ha desarrollado un espectacular crecimiento, de tal forma que, actualmente, estos tratamientos son utilizados como acabado final de herramientas y piezas de distintos procesos industriales. Entre las distintas aplicaciones de los recubrimientos por PVD cabe destacar los recubrimientos cerámicos ultraduros, aplicados a herramientas de corte y mecanizado de aceros rápidos y carburo de tungsteno Co-cementado. Es en este tipo de aplicación donde mayores esfuerzos se han invertido, existiendo en la actualidad un menú de recubrimientos (TiN, AlTiN, CrN, ZrN, DLC...) donde poder seleccionar el más adecuado para una aplicación concreta (1). Aunque estas soluciones pueden mejorar el funcionamiento y alargar en gran medida la vida útil de herramientas, el elevado coste de las mismas, así como otros factores de los procesos productivos (tiempos muertos, perdidas por no-calidad...) fomentan la demanda de recubrimientos más resistentes y con mejores propiedades. Actualmente las dos grande líneas de investigación en recubrimientos ultraduros por PVD se centran en capas nanoestructuradas y en multicapas. En esta dirección en este trabajo se investigan y comparan las propiedades mecánicas superficiales de multicapas TiN/CrN y TiN/AlTiN depositadas sobre substratos de WC Co-cementado.

\section{EXPERIMENTAL}

\subsection{Preparación de muestras}

Discos de $30 \mathrm{~mm}$ de diámetro y $6 \mathrm{~mm}$ de espesor de WC Co-cementado fueron sometidos a un baño ultrasónico de acetona durante 15 minutos antes de ser recubiertos. Los recubrimientos fueron realizados mediante arco eléctrico reactivo (CVAE) en un equipo METAPLAS
MZR323. Este equipo dispone de seis evaporadores que trabajan a 30V y 100 A con un campo magnético débil. Antes del proceso de deposición se llevó a cabo una limpieza iónica (AEGD-Arc Enhaced Glow Discharge) bombardeando el substrato con argón durante 15 minutos, eliminando la contaminación superficial. Este sistema disminuye en gran medida la aparición de las microgotas típicas de los procesos de arco eléctrico (2). Los recubrimientos se llevaron a cabo a una temperatura aproximada de $450^{\circ} \mathrm{C}$ empleando nitrógeno como gas reactivo. Los niveles de vacío fueron de $10^{-4} \mathrm{~Pa}$ de vacío previo y $10^{-1} \mathrm{~Pa}$ de presión de trabajo.

\subsection{Caracterización mecánica superficial}

La dureza Universal H.U. (dureza bajo carga con indentador Vickers) de los materiales recubiertos y de las muestra de referencia se han medido con un Microdurómetro FICHERSCOPE H100 empleando una carga final de $10 \mathrm{mN}(3,4)$. El equipo permite además calcular algunas de las propiedades elástico-plásticas superficiales más interesantes como el Módulo de Elasticidad y el tanto por ciento de recuperación elástica. Los ensayos de fricción se han realizado utilizando un Tribómetro FALEX ISC-320PC, con una configuración ballon-disk que permite controlar todos los parámetros relevantes, incluida la humedad relativa. Para los ensayos se emplearon bolas de $1 / 2$ " de WC Co-cementado. El coeficiente de desgaste se calculó midiendo el volumen perdido en las trazas de los ensayos de fricción (5) mediante un perfilómetro interferométrico WYCO RST $500(6,9)$. 


\section{RESULTADOS}

La figura $1 \mathrm{a}-\mathrm{b}$ muestra las curvas de carga y descarga a $10 \mathrm{mN}$ de carga final para los dos tipos de multicapas y para las muestra de referencia sin recubrir. En estas gráficas es posible observar un ligero aumento de la dureza tanto para la multicapa $\mathrm{TiN} / \mathrm{CrN}$ como para la TiN/AlTiN, así como una elevada dispersión debido a la rugosidad de los substratos. Por otro lado, también en la figura $1 \mathrm{c}-\mathrm{d}$ se recogen los perfiles de dureza hasta un carga final de $750 \mathrm{mN}$ de las muestras recubiertas y de las muestras de referencia. A medida que aumenta la carga final se observa una disminución de la dispersión debido a que al aumentar la profundidad del indentador los efectos de la rugosidad son menos importantes. También puede observarse en las gráficas 1 c-d que aunque a medida que penetra el indentador disminuye la dureza de los recubrimientos, a más de 1 micra todavía pueden observarse claros aumentos de dureza en los recubrimientos con respecto a las muestras sin recubrir. Los ensayos de fricción revelaron diferencias apreciables entre los dos tipos de recubrimientos: mientras que para las multicapas TiN/AlTiN el coeficiente de fricción oscilaba entre 0.70.8, para las multicapas TiN/CrN el coeficiente oscilaba entre 0.4-0.6 (tabla I). Por otro lado y como puede observarse en la tabla I y en la figura 2, el coeficiente de desgaste para las multicapas TiN/Cr fue más de 4 veces menor que para los recubrimientos TiN/AlTiN.

TABLA I.

\begin{tabular}{|c|c|c|}
\hline & TiN/CrN & TiN/AlTiN \\
\hline $\mathrm{E} /\left(1-\mathrm{v}^{2}\right)(10 \mathrm{mN})$ & $417 \pm 8 \mathrm{GPa}$ & $500 \pm 11 \mathrm{GPa}$ \\
\hline$\%$ We $(10 \mathrm{mN})$ & 54.5 & 53.6 \\
\hline Coef. de Fricción & $0.4 \rightarrow 0.6$ & $0.7 \rightarrow 0.8$ \\
\hline Coef. de Desgaste $\left(\mathrm{N}^{-1} \mathrm{~m}^{2}\right)$ & $1.52 \mathrm{E}-15$ & $6,37 \mathrm{E}-15$ \\
\hline
\end{tabular}

\section{CONCLUSIONES}

Las multicapas TiN/CrN y TiN/AlTiN muestran microdurezas bajo carga de más de $15.000 \mathrm{MPa}$ superiores en más de un $20 \%$ a la dureza del substrato WC Co-cementado (12.500 MPa).

Los coeficientes de fricción de las muestras recubiertas van desde 0.7-0.8 para las multicapas de TiN/ AlTiN hasta 0.4-0.6 para las multicapas de TiN/CrN. Estos coeficientes son superiores a los coeficientes de fricción mostrados por el substrato sin recubrir (0.4).

La multicapa TiN/CrN se mostró 4 veces más resistente al desgaste, con un coeficiente de $1.510^{-15} \mathrm{~N}^{-1} \mathrm{~m}^{2}$, frente al coeficiente de 6.4 $10^{-15} \mathrm{~N}^{-1} \mathrm{~m}^{2}$ mostrado por la muestra de TiN/AlTiN.

\section{AGRADECIMIENTOS}

Los autores desean expresar su agradecimiento al Gobierno de Navarra por el soporte dado para la realización de este trabajo mediante el proyecto TRIBOTEC (financiado por el protocolo de colaboración Aquitania-Navarra).

\section{BIBLIOGRAFÍA}

1. J. Vetter, A.J. Perry, "Advances in cathodic arc technology using electrons extracted from the vacuum arc". Surf. Coat. Technol. 61 (1993), 305-309.

2. A.T. Alpas, Z.N. Farhat, Y. Ding, D.O. Northwood, "Effect of grain size on friction and wear of nanocristalline aluminium". Mater. Sci Eng A206 (1996), 302-313.
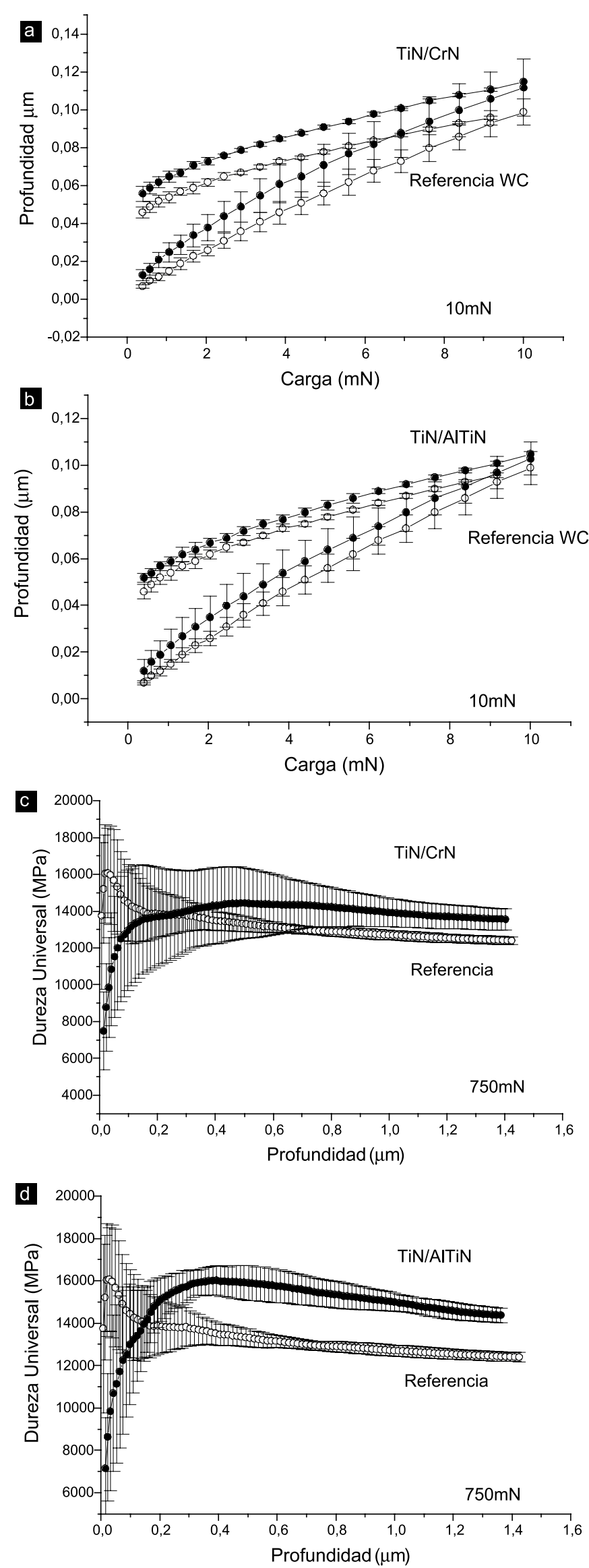

Figura 1. Curvas de carga descarga a $10 \mathrm{mN}$ de carga final para las muestras recubiertas (a) y para las referencias (b); Curvas de dureza frente a profundidad para las muestras recubiertas (c) y referencias (d). 

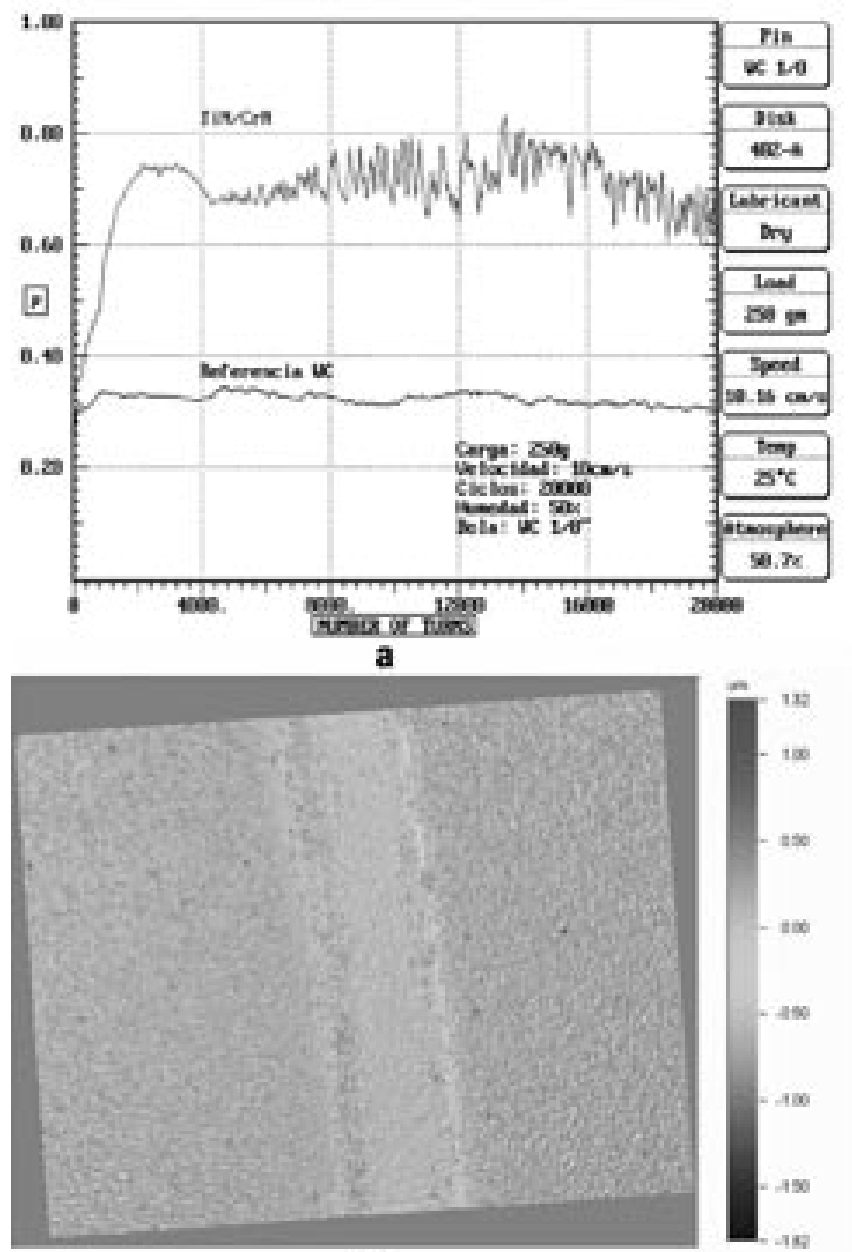

C

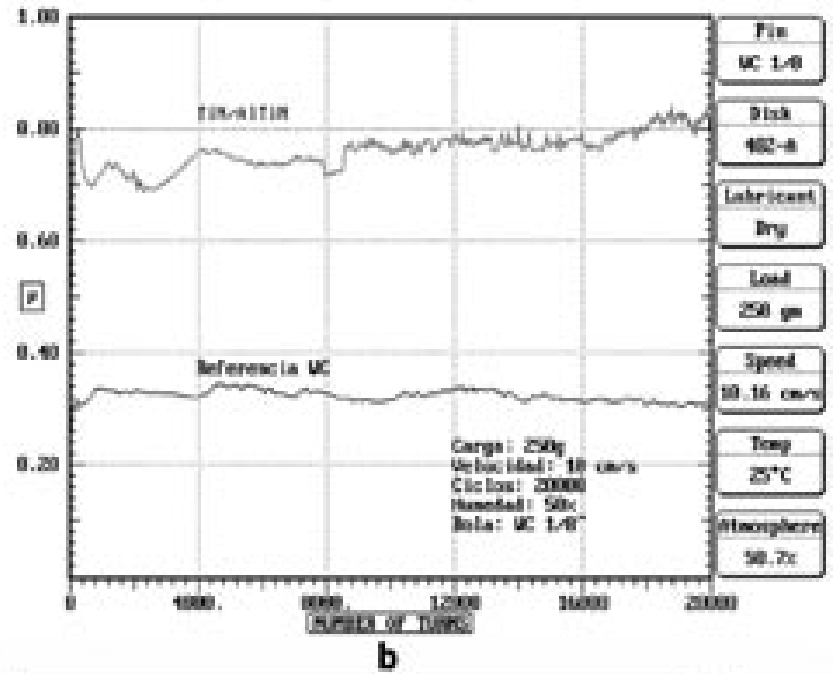

b

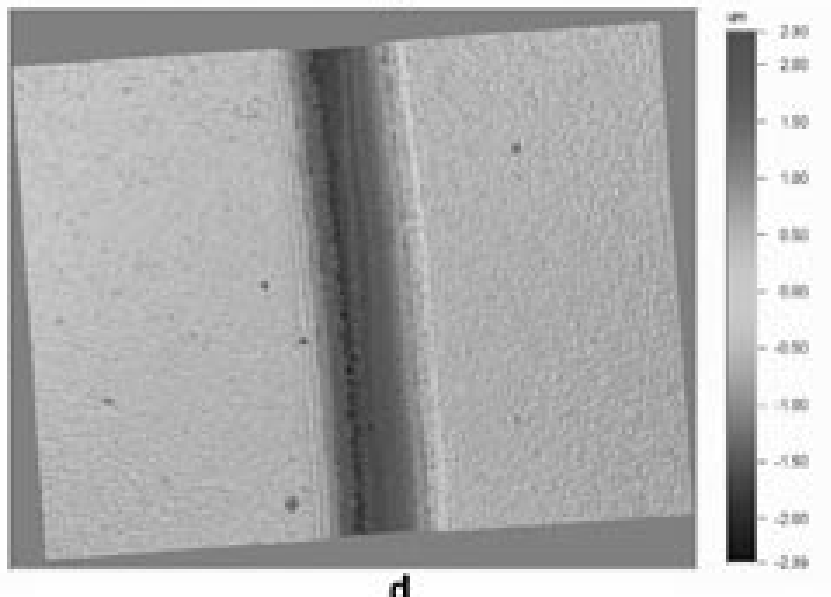

Figura 2. Evolución del coeficiente de fricción comparado con la referencia para las multicapas TiN/CrN (a) y para TiN/AlTiN (b); Surcos de desgaste para la muestra de TiN/CrN (c) y para la muestra TiN/AlTiN (d).

3. J.L. Loubet, J.M. Georges, G. Meille, "Vickers Indentation Curves of Elastoplastic Materilas". Micr. Techn. in Mater. Sci. and Engin. ASTM STP 889 (1986), 72-89.

4. W.C. Oliver, G.M., Pharr, "An Improved Technique for Determining Hardness and Elastic Modulus Using Load and Displacement Sensing Indentation Experiments". J. Mater. Res. 7 (1992), 1564-1583

5. H. Herz, "Uber die Ber" uhrung fester elasticher K"orper". J. reine und angewandte Mathematik 92 (1882), 156
6. Standard test method for wear testing with a Pin-on-Disk Apparatus, ASTM G99-95 A.

7. P.J. Caber, S.J. Martinek, R. J. Niemann, "A new Interferometric Profiler for Smooth and Rough Surfaces". Proceedings SPIE (1993) 2088

8. J.C. Wyant, "How to Extend Interferometric from Rough-Surface Tests". Laser Focus World (1993), 131-133

9. P.J. Caber, "Interferometric Profiler for Rough Surfaces". Appl. Opt. 32(19) (1993), 3438-3441

Recibido: 1.2 .03

Aceptado: 30.11 .03 